\title{
Hamiltonian approach to the transport properties of superconducting quantum point contacts
}

\author{
J. C. Cuevas, A. Martín-Rodero and A. Levy Yeyati \\ Departamento de Física Teórica de la Materia Condensada $C$-V. \\ Facultad de Ciencias. Universidad Autónoma de Madrid. \\ E-28049 Madrid. Spain.
}

\begin{abstract}
A microscopic theory of the transport properties of quantum point contacts giving a unified description of the normal conductor-superconductor (N-S) and superconductor-superconductor (S-S) cases is presented. It is based on a model Hamiltonian describing charge transfer processes in the contact region and makes use of non-equilibrium Green function techniques for the calculation of the relevant quantities. It is explicitly shown that when calculations are performed up to infinite order in the coupling between the electrodes, the theory contains all known results predicted by the more usual scattering approach for N-S and S-S contacts. For the latter we introduce a specific formulation for dealing with the non-stationary transport properties. An efficient algorithm is developed for obtaining the dc and ac current components, which allows a detailed analysis of the different current-voltage characteristics for all range of parameters. We finally address the less understood small bias limit, for which some analytical results can be obtained within the present formalism. It is shown that four different physical regimes can be reached in this limit depending on the values of the inelastic scattering rate and the contact transmission. The behavior of the system in these regimes is discussed together with the conditions for their experimental observability.
\end{abstract}


PACS numbers: 74.50.+r, 85.25.Cp, 73.20.Dx

\section{INTRODUCTION}

Electronic transport through N-S and S-S junctions and weak links has been the object of interest for many years [1.2]. Much of the theoretical understanding of these systems has been obtained by analyzing simple models where a one-dimensional character is assumed [2]:3]. These kind of models have been very useful for clarifying the microscopic origin of basic phenomena like the excess current in N-S and S-S contacts and the subgap structure in S-S and S-Insulator-S (S-I-S) junctions. In particular, the one-dimensional scattering model introduced by Blonder et al. [3] (hereafter referred to as BTK) has provided a simple way of analyzing the transport properties in terms of normal electron transmission and Andreev reflection probabilities.

With the recent advances in the fabrication of nanoscale devices [4, [5], a closer comparison between the predictions of simple quasi one-dimensional theories and experimental results is now at hand. Refs. [4.5 provide two different examples of the progress made in the experimental achievement of a superconducting quantum point-contact (SQPC). This possibility has provoked a renewed interest in more detailed and quantitative analysis of models involving a few conducting channels. It turns out that, in spite of its apparent simplicity, the case of a single channel SQPC still contains non-trivial physical behavior at certain

regimes. Single mode contacts have been analyzed recently by different authors [6 8] who have obtained new quantitative results for the case of small applied voltage where multiple Andreev reflections (MAR) play a crucial role.

Some of these recent works [7,8] illustrate the existence of a variety of regimes controlled by the contact transmission and quasiparticle damping in the small bias limit. A full understanding of the different regimes, together with the transitions between them remains to be explored. This will be thoroughly analyzed in this paper. 
Traditionally, quantum transport in microelectronic devices has been mainly addressed by two different approaches: one is based on the scattering picture first introduced by Landauer [9] in which the transport properties are expressed in terms of transmission and reflection scattering amplitudes. This approach is generally used in a phenomenological way by replacing the device with a simple scattering structure. The natural extension of this picture to the superconducting case was provided by the already mentioned BTK model.

A different point of view arises when the problem is analyzed starting from a microscopic Hamiltonian. We shall very generally call "Hamiltonian approach" the theories which take this starting point. The origin of this approach can be traced back to the work by Bardeen who introduced the tunnel Hamiltonian approximation for describing a tunnel junction [10]. Most of the calculations based on this tunnel Hamiltonian were restricted to the lowest order transport processes like in the calculation of the Josephson current in a S-I-S junction [11]. Multi-particle tunneling processes (MPT) were first discussed by Schrieffer and Wilkins [12] as a possible explanation for the observed subgap structure in superconducting tunnel junctions. The contributions of these higher order processes were found to be divergent, which has led to the quite extended belief that the Hamiltonian approach is pathological except for describing the lowest order tunneling processes.

One of the aims of the present work is to show that starting from a very simple model Hamiltonian describing a single channel contact it is possible to obtain results for N-S and S-S contacts in agreement with those provided by scattering theory. As will be shown below inclusion of higher order processes up to infinite order eliminates the pathologies associated with finite order perturbation theory.

On the other hand, the Hamiltonian approach in combination with non-equilibrium Green function techniques presents its own advantages. In recent publications we have shown how this approach can be generalized for dealing with situations where self-consistency of the superconducting order parameter is needed [13]. Moreover, the formulation in terms of Green functions is specially well suited for dealing with correlation effects when strong e-e interactions are present [14]. 
The paper is organized as follows: In section II the general model for a superconducting weak link in a local representation is introduced. In particular, we discuss how this model can be simplified in order to describe a single mode contact. We then introduce the nonequilibrium Green function formalism by means of which the total current through the contact can be expressed. In section III we analyze the more simple N-N and N-S contacts. The study of the N-N case allows us to define the contact transmission coefficient in terms of the microscopic parameters of our model. The complete correspondence with the results

of the BTK scattering theory is then established by analyzing the N-S contact. For this last case we derive an analytical expression for the total current as a function of the contact transmission. Section IV is devoted to the S-S case. We first show how the problem of the calculation of the ac current components can be reduced to the evaluation of an algebraic set of linear equations. This allows us to study in detail the general features of the dc and ac $I-V$ characteristics for the whole range of voltages and transmissions. We finally concentrate on the limit $V \ll \Delta$ where the more interesting and less understood physics takes place. Section $\mathrm{V}$ is devoted to some concluding remarks.

\section{MODEL AND METHOD}

In this paper we shall consider "point contact-like" geometries consisting very generally of two wide (3D or 2D) electrodes connected by a narrow constriction. The constriction length, $L_{C}$, is assumed to be much smaller than the superconducting coherence length and its width, $W_{C}$, comparable to the Fermi wavelength, $\lambda_{F}$. For a sufficiently short constriction the detailed form of the self-consistent order parameter and electrostatic potential in the region between the electrodes becomes irrelevant [13] allowing us to represent them by simple step functions. On the other hand, the condition $W_{C} \sim \lambda_{F}$ implies that there is only a reduced number of quantum transverse channels through the constriction. One can think of different experimental realizations of such a physical situation, like for instance the 
recently developed atomic size break-junctions [4] and the split-gate S-2DEG-S SQPC of Takayanagi et al. [0]. These two situations are schematically represented in Fig. 1.

The analysis of the transport and electronic properties of such a system would involve, for the most general case where both electrodes are superconductors, the solution of the time-dependent Bogoliubov-de Gennes (BdeG) equations [15]. In previous works we have developed an approach to these kind of problems in which the BdeG equations are formulated in a site representation [13]. This representation can be viewed either as a tight-binding description of the electronic states or as a discretization of the BdeG equations. The first case would be more suitable for describing systems like an atomic size contact (Fig. 1 a), while the second could be used to represent constrictions involving a 2DEG in semiconducting heterostructures (Fig. 1 b). In the absence of applied fields the mean-field Hamiltonian giving rise to the BdeG equations would take the form

$$
\hat{H}=\sum_{i, \sigma}\left(\epsilon_{i}-\mu\right) c_{i \sigma}^{\dagger} c_{i \sigma}+\sum_{i \neq j, \sigma} t_{i j} c_{i}^{\dagger} c_{j}+\sum_{i}\left(\Delta_{i}^{*} c_{i \downarrow}^{\dagger} c_{i \uparrow}^{\dagger}+\Delta_{i} c_{i \uparrow} c_{i \downarrow}\right)
$$

where $i, j$ run over the sites used to represent the system. By appropriately choosing the different parameters $\epsilon_{i}, t_{i j}$ and $\Delta_{i}$ one can model different junction geometries [13]. In order to analyze the case of biased contacts it is convenient to simplify this model Hamiltonian taking the step-like behavior of the order parameter and electrostatic potentials in a point contact geometry into account. To do this we take the complex order parameter and the chemical potentials as constants on the left and right electrodes (denoted by $\left(\Delta_{L}, \mu_{L}\right)$ and $\left(\Delta_{R}, \mu_{R}\right)$ respectively). The case of a single quantum channel connecting both electrodes can then be described by the following Hamiltonian

$$
\hat{H}=\hat{H}_{L}+\hat{H}_{R}+\sum_{\sigma}\left(t c_{L \sigma}^{\dagger} c_{R \sigma}+t^{*} c_{R \sigma}^{\dagger} c_{L \sigma}\right)-\mu_{L} \hat{N}_{L}-\mu_{R} \hat{N}_{R}
$$

where $\hat{H}_{L}$ and $\hat{H}_{R}$ correspond to the uncoupled electrodes while the hopping term describes the electron transfer processes between the outermost sites on both electrodes. We would like to stress that, although this is a very simple model Hamiltonian (formally equivalent to a tunnel Hamiltonian), it contains the relevant physics of a quantum point contact which 
depends essentially on the contact normal transmission coefficient. In our model this transmission can vary between $\sim 0$ (tunnel limit) and $\sim 1$ (ballistic regime) as a function of the coupling parameter $t$. This will be discussed in section III.

For the case of a symmetric superconducting contact in the presence of an applied bias voltage $e V=\mu_{L}-\mu_{R}$ it is convenient to perform a gauge transformation [16] by means of which Hamiltonian (2) adopts the following time-dependent form

$$
\hat{H}(\tau)=\hat{H}_{L}+\hat{H}_{R}+\sum_{\sigma}\left(t e^{i \phi(\tau) / 2} c_{L \sigma}^{\dagger} c_{R \sigma}+t^{*} e^{-i \phi(\tau) / 2} c_{R \sigma}^{\dagger} c_{L \sigma}\right)
$$

where the superconducting phase difference $\phi(\tau)=\phi_{0}+2 e V \tau / \hbar$ appears as a phase factor in the hopping elements.

\section{A. Current in terms of the non-equilibrium Green functions}

Within the previously introduced model, the average total current through the contact is given by

$$
I(\tau)=\frac{i e}{\hbar} \sum_{\sigma}\left(t<c_{L \sigma}^{\dagger}(\tau) c_{R \sigma}(\tau)>-t^{*}<c_{R \sigma}^{\dagger}(\tau) c_{L \sigma}(\tau)>\right)
$$

where, depending on the gauge choice, $t$ can include a time dependent phase like in Eq. (3). The averaged quantities in Eq. (4) can be expressed in terms of non-equilibrium Keldysh Green functions $\hat{G}^{+-}$17]. For the superconducting state it is convenient to introduce the $2 \times 2$ Nambu representation in which $\hat{G}_{i, j}^{+-}$adopts the form 13

$$
\hat{G}_{i, j}^{+-}\left(\tau, \tau^{\prime}\right)=i\left(\begin{array}{l}
<c_{j \uparrow}^{\dagger}\left(\tau^{\prime}\right) c_{i \uparrow}(\tau)><c_{j \downarrow}\left(\tau^{\prime}\right) c_{i \uparrow}(\tau)> \\
<c_{j \uparrow}^{\dagger}\left(\tau^{\prime}\right) c_{i \downarrow}^{\dagger}(\tau)><c_{j \downarrow}\left(\tau^{\prime}\right) c_{i \downarrow}^{\dagger}(\tau)>
\end{array}\right) .
$$

In terms of the $\hat{G}^{+-}$, the current is given by

$$
I(\tau)=\frac{2 e}{\hbar}\left[\hat{t} \hat{G}_{R L}^{+-}(\tau, \tau)-\hat{t}^{*} \hat{G}_{L R}^{+-}(\tau, \tau)\right]_{11},
$$

where $\hat{t}$ is the Nambu representation of the hopping elements 


$$
\hat{t}=\left(\begin{array}{cc}
t & 0 \\
0 & -t^{*}
\end{array}\right) .
$$

The problem then consists in the determination of these non-equilibrium Green functions $\hat{G}^{+-}$. This can be formally achieved by treating the coupling $\hat{t}$ as a perturbation. The unperturbed Green functions correspond to the uncoupled electrodes in equilibrium. For a symmetric contact, and neglecting finite band-width effects, the uncoupled retarded and advanced Green functions can be expressed as

$$
\hat{g}_{L L}^{r, a}(\omega)=\hat{g}_{R R}^{r, a}(\omega)=\frac{1}{W \sqrt{\Delta^{2}-(\omega \pm i \eta)^{2}}}\left(\begin{array}{cc}
-\omega \pm i \eta & \Delta \\
\Delta & -\omega \pm i \eta
\end{array}\right),
$$

where $W$ is an energy scale related to the normal density of states at the Fermi level by $\rho\left(\epsilon_{F}\right) \sim 1 /(\pi W)$ and $\eta$ is a small energy relaxation rate that takes into account the damping of the quasi-particle states due to inelastic processes inside the electrodes [18]. On the other hand, the unperturbed $\hat{g}^{+-}(\omega)$ satisfy the relation

$$
\hat{g}^{+-}(\omega)=2 \pi i \hat{\rho}(\omega) n_{F}(\omega)
$$

where $\hat{\rho}(\omega)=(1 / \pi) \operatorname{Im}\left[\hat{g}^{a}(\omega)\right]$ and $n_{F}(\omega)$ is the Fermi function.

For the coupled system, the functions $\hat{G}_{i, j}^{+-}$can be obtained from the retarded and advanced functions by means of the integral equation

$$
\begin{aligned}
\hat{G}^{+-}\left(\tau, \tau^{\prime}\right)= & \int d \tau_{1} d \tau_{2}\left[\hat{I} \delta\left(\tau-\tau_{1}\right)+\hat{G}^{r}\left(\tau, \tau_{1}\right) \hat{\Sigma}^{r}\left(\tau_{1}\right)\right] \hat{g}^{+-}\left(\tau_{1}-\tau_{2}\right) \times \\
& {\left[\hat{I} \delta\left(\tau_{2}-\tau^{\prime}\right)+\hat{\Sigma}^{a}\left(\tau_{2}\right) \hat{G}^{a}\left(\tau_{2}, \tau^{\prime}\right)\right], }
\end{aligned}
$$

where $\hat{G}^{r, a}$ can be derived from their corresponding Dyson equations

$$
\hat{G}^{r, a}\left(\tau, \tau^{\prime}\right)=\hat{g}^{r, a}\left(\tau-\tau^{\prime}\right)+\int d \tau_{1} \hat{g}^{r, a}\left(\tau-\tau_{1}\right) \hat{\Sigma}^{r, a}\left(\tau_{1}\right) \hat{G}^{r, a}\left(\tau_{1}, \tau^{\prime}\right)
$$

In the above equations the self-energies take the simple form $\hat{\Sigma}_{L L}^{r, a}=\hat{\Sigma}_{R R}^{r, a}=0$ and $\hat{\Sigma}_{L R}^{r, a}=$ $\left(\hat{\Sigma}_{R L}^{r, a}\right)^{*}=\hat{t}$.

In sections III and IV details of the solution of these integral equations are given. 


\section{N-N AND N-S CONTACTS}

In this section we briefly review the N-N and N-S cases. The analysis of the N-N case permits to define the normal transmission coefficient of the contact in terms of the microscopic parameters of the model, which is necessary for making contact with the scattering approach. The equivalence of both approaches is illustrated by comparing our results for the N-S contact with those of the BTK model [3].

As we shall deal with a stationary situation it is convenient to adopt the time-independent formulation based on Hamiltonian (2). In this case the Green functions depend on the difference of its temporal arguments and integral equations (10) and (11) become simple algebraic equations when Fourier transformed. Then we have

$$
\begin{gathered}
\hat{G}^{r, a}(\omega)=\hat{g}^{r, a}(\omega)+\hat{g}^{r, a}(\omega) \hat{\Sigma}^{r, a}(\omega) \hat{G}^{r, a}(\omega) \\
\hat{G}^{(+-),(-+)}(\omega)=\left[\hat{I}+\hat{G}^{r}(\omega) \hat{\Sigma}^{r}(\omega)\right] \hat{g}^{(+-),(-+)}(\omega)\left[\hat{I}+\hat{\Sigma}^{a}(\omega) \hat{G}^{a}(\omega)\right] .
\end{gathered}
$$

where $\hat{g}^{-+}(\omega)=-2 \pi i \hat{\rho}(\omega)\left[1-n_{F}(\omega)\right]$. On the other hand, it is convenient to rewrite expression (6) for the current as [13, 19]

$$
I=\frac{2 e}{h} t^{2} \int_{-\infty}^{\infty}\left[g_{L L, 11}^{+-} G_{R R, 11}^{-+}(\omega)-g_{L L, 11}^{-+} G_{R R, 11}^{+-}(\omega)\right] d \omega
$$

where we have assumed that the left electrode is in the normal state, while the right one can be either normal or superconducting.

Let us first analyze the case where both electrodes are in the normal state. Details on

the calculations of the Green functions for this case have been given elsewhere [20]. As in the scattering approach, the current can be written as

$$
I=\frac{2 e}{h} \int_{-\infty}^{\infty} T(\omega, V)\left[n_{F}(\omega-e V)-n_{F}(\omega)\right] d \omega
$$

where $T(\omega, V)$ is an energy-dependent transmission coefficient which is given by

$$
T(\omega, V)=\frac{4 \pi^{2} t^{2} \rho_{L L}(\omega-e V) \rho_{R R}(\omega)}{\left|1-t^{2} g_{L L}(\omega-e V) g_{R R}(\omega)\right|^{2}} .
$$


This expression can be further simplified assuming that the normal state spectral densities are constant over an energy scale $W \gg V, \Delta$. This is consistent with the assumptions leading to Eq. (8). Within this energy scale the coefficient $T(\omega, V)$ becomes an energyindependent quantity $\alpha$

$$
T(\omega, V) \simeq \frac{4 t^{2} / W^{2}}{\left(1+t^{2} / W^{2}\right)^{2}} \equiv \alpha .
$$

The normal linear conductance of this single mode contact is then given by the Landauer formula $G_{N N}=\left(2 e^{2} / h\right) \alpha$. Notice that $\alpha$ can vary between zero and one as a function of $t$. The $\alpha \rightarrow 0$ limit is reached both for $t / W \ll 1$ and for $t / W \gg 1$, while the ballistic limit, i.e. $\alpha \sim 1$, is reached when $t / W \sim 1$.

It is worth to further clarify the role of the hybridization parameter $t$ within our point contact model. Although in the context of the tunnel Hamiltonian approach it has been customary to identify $\sim t^{2}$ with the transmission probability, this strictly holds for the tunnel regime (lowest order perturbation theory in $t$ ). However, the actual expression for the contact transparency (Eq. (17)) including all order processes is a non-linear function of $t^{2}$. While the ballistic condition is achieved for $t^{2} / W^{2} \sim 1$, the way in which $\alpha$ approaches unity is completely different from $\sim t^{2} / W^{2}$, as can be seen from Eq. (17).

In the N-S case $\left(\Delta_{L}=0, \Delta_{R}=\Delta\right)$ starting from Eq. (12) and (13) after some simple algebra we obtain the following expression for the current as the sum of four different contributions $I=I_{1}+I_{2}+I_{3}+I_{A}$, where

$$
\begin{aligned}
& I_{1}=\frac{8 e}{h} \pi^{2} t^{2} \int_{-\infty}^{\infty} d \omega\left|1+t G_{R L, 11}^{r}(\omega)\right|^{2} \rho_{L L, 11}(\omega-e V) \rho_{R R, 11}(\omega)\left[n_{F}(\omega-e V)-n_{F}(\omega)\right] \\
& I_{2}=-\frac{16 e}{h} \pi^{2} t^{2} \int_{-\infty}^{\infty} d \omega R e\left\{t G_{L R, 21}^{a}(\omega)\left[1+t G_{R L, 11}^{r}(\omega)\right]\right\} \times \\
& \rho_{L L, 11}(\omega-e V) \rho_{R R, 12}(\omega)\left[n_{F}(\omega-e V)-n_{F}(\omega)\right] \\
& I_{3}=\frac{8 e}{h} \pi^{2} t^{4} \int_{-\infty}^{\infty} d \omega\left|G_{R L, 12}(\omega)\right|^{2} \rho_{L L, 11}(\omega-e V) \rho_{R R, 22}(\omega)\left[n_{F}(\omega-e V)-n_{F}(\omega)\right] \\
& I_{A}=\frac{8 e}{h} \pi^{2} t^{4} \int_{-\infty}^{\infty} d \omega\left|G_{R R, 12}(\omega)\right|^{2} \rho_{L L, 11}(\omega-e V) \rho_{L L, 22}(\omega+e V)\left[n_{F}(\omega-e V)-n_{F}(\omega+e V)\right] .
\end{aligned}
$$

Written in this form, each contribution has a clear interpretation in terms of elementary 
processes that can be identified by inspection of the intervening spectral densities. Although there is a formal resemblance of the above expressions with those of tunnel theory [21] Eq. (18) contains all possible processes up to infinite order in $t$. Thus, $I_{1}$ corresponds to normal electron transfer between the electrodes, while $I_{2}$ corresponds also to a net transfer of a single electron with creation or annihilation of pairs as an intermediate state. On the other hand, $I_{3}$ arises from processes where an electron in the normal electrode is converted into a hole in the superconducting side, i.e. processes with "branch crossing" in the BTK language. Finally, $I_{A}$ arises from Andreev reflection processes in which an electron (with an associated spectral weight $\left.\rho_{L L, 11}(\omega-e V)\right)$ is transmited from the left to the right electrode with a hole reflecting backwards into the normal electrode (with an associated spectral weight $\left.\rho_{L L, 22}(\omega+e V)\right)$ while a Cooper pair is created in the superconducting side with a probability proportional to $\left|G_{R R, 12}(\omega)\right|^{2}$.

As expected, the only non-zero contribution for $\mathrm{eV}<\Delta$ is $I_{A}$, while all processes contribute for $\mathrm{eV}>\Delta$. With the same simplifying assumptions leading to Eq. (17) the differential conductance at zero temperature adopts the simple form

$$
\begin{array}{ll}
G_{N S}(V)=\frac{4 e^{2}}{h} \frac{\alpha^{2}}{(2-\alpha)^{2}-4(1-\alpha)\left(\frac{e V}{\Delta}\right)^{2}} ; \quad e V \leq \Delta \\
G_{N S}(V)=\frac{4 e^{2}}{h} \frac{\alpha}{\alpha+(2-\alpha) \sqrt{1-\left(\frac{\Delta}{e V}\right)^{2}}} ; \quad e V>\Delta .
\end{array}
$$

This expression can be shown to be equivalent to the one obtained from the BTK model [3] with the correspondence $Z=\left[1-(t / W)^{2}\right] /(2 t / W)$, where $Z$ is the dimensionless phenomenological parameter controlling the barrier height in the BTK model.

It is worth noticing that the differential conductance rises from $\left(4 e^{2} / h\right) \alpha^{2} /(2-\alpha)^{2}$ at $V=$ 0 to the value $4 e^{2} / h$ at $e V=\Delta$, this last value being independent of the contact transmission. This result can never be obtained within any finite order perturbative approximation in $t$, but requires an infinite order calculation [22]. This sort of non-perturbative features are also very important in the S-S case [23], as will be discussed in the next section.

Finally, it is possible to obtain from Eq. (18) an analytical expression of the total current at zero temperature. For $\mathrm{eV}>\Delta$ this can be written as $I=I_{1}+I_{2}$ with 


$$
\begin{aligned}
I_{1}= & \frac{e \Delta}{h} \frac{\alpha^{2}}{(2-\alpha) \sqrt{1-\alpha}} \ln \left[\frac{1+\left[\frac{2 \sqrt{1-\alpha}}{2-\alpha}\right]}{1-\left[\frac{2 \sqrt{1-\alpha}}{2-\alpha}\right]}\right] \\
I_{2}(x)= & \frac{4 e}{h} \Delta\left[\frac{\alpha^{2}}{4(1-\alpha)}+\frac{\alpha}{x\left[\alpha+(2-\alpha) \sqrt{1-x^{2}}\right]}-\frac{\alpha(2-\alpha)^{2} x}{4(1-\alpha)\left[\alpha+(2-\alpha) \sqrt{1-x^{2}}\right]}\right. \\
& \left.+\frac{\alpha^{2}(2-\alpha)}{8(1-\alpha)^{3 / 2}} \ln \left[\frac{\left(\frac{x}{1+\sqrt{1-x^{2}}}+\frac{1}{\sqrt{1-\alpha}}\right)\left(1-\frac{1}{\sqrt{1-\alpha}}\right)}{\left(\frac{x}{1+\sqrt{1-x^{2}}}-\frac{1}{\sqrt{1-\alpha}}\right)\left(1+\frac{1}{\sqrt{1-\alpha}}\right)}\right]\right],
\end{aligned}
$$

where $I_{1}$ is the gap contribution to the total current, $I_{2}$ is the contribution coming from energies outside the gap and $x=\Delta / e V$. This allows to analyze with detail the "excess" current, defined as $I_{e x c}=\lim _{V \rightarrow \infty}\left(I_{N S}-I_{N N}\right)$, as a function of the contact transparency. We find $I_{e x c}=I_{e x c_{1}}+I_{e x c_{2}}$

$$
\begin{aligned}
& I_{e x c_{1}}=\frac{e \Delta}{h} \frac{\alpha^{2}}{(2-\alpha) \sqrt{1-\alpha}} \ln \left[\frac{1+\left[\frac{2 \sqrt{1-\alpha}}{2-\alpha}\right]}{1-\left[\frac{2 \sqrt{1-\alpha}}{2-\alpha}\right]}\right] \\
& I_{e x c_{2}}=\frac{e \Delta}{h} \alpha^{2}\left[\frac{1}{1-\alpha}+\frac{2-\alpha}{2(1-\alpha)^{3 / 2}} \ln \left[\frac{1-\sqrt{1-\alpha}}{1+\sqrt{1-\alpha}}\right]\right],
\end{aligned}
$$

where $I_{e x c_{1}}$ and $I_{e x c_{2}}$ are respectively the contributions coming from energies inside and outside the gap. As can be easily checked, $I_{\text {exc } 1}>0$ while $I_{e x c_{2}}<0$ the total excess current being always positive. For $\alpha=1$ the well known result [24,28] for the ballistic contact $I_{e x c}=(8 / 3) e \Delta / h$ is recovered.

\section{THE S-S CONTACT}

\section{A. An efficient algorithm for evaluating the ac current}

As commented in section II, for the case of a voltage-biased S-S contact it is convenient to start from Hamiltonian (3) in which the applied bias is taken into account through a time-dependent phase factor in the hopping element, which in a Nambu representation has the form

$$
\hat{t}=\left(\begin{array}{cc}
t e^{i \phi(\tau) / 2} & 0 \\
0 & -t e^{-i \phi(\tau) / 2}
\end{array}\right)
$$


where $\phi=\phi_{0}+2 e V \tau / \hbar$ is the time-dependent superconducting phase difference.

This explicit time dependence indicates that all dynamic quantities can be expanded as Fourier series in all possible harmonics of the fundamental frequency $\omega_{0}=2 e V / \hbar$ [7]. For instance, the total current can be written as

$$
I(\tau)=\sum_{m} I_{m} e^{i m \omega_{0} \tau}
$$

We shall now show how these Fourier coefficients, $I_{m}$, can be efficiently evaluated within the non-equilibrium Green function formalism. Let us first notice that the non-equilibrium Green functions appearing in Eqs. (10) and (11) do not depend only on the difference of their temporal arguments, and have therefore a generalized Fourier expansion of the form 25,26

$$
\hat{G}\left(\tau, \tau^{\prime}\right)=\frac{1}{2 \pi} \sum_{n} \int d \omega e^{-i \omega \tau} e^{i\left(\omega+n \omega_{0} / 2\right) \tau^{\prime}} \hat{G}\left(\omega, \omega+n \omega_{0} / 2\right) .
$$

Hereafter we shall use the notation $\hat{G}_{n m}(\omega)=\hat{G}\left(\omega+n \omega_{0} / 2, \omega+m \omega_{0} / 2\right)$. Different Fourier components $\hat{G}_{n m}$ are related by $\hat{G}_{n m}(\omega)=\hat{G}_{n-m, 0}\left(\omega+m \omega_{0} / 2\right)$. For the particular gauge choice adopted here, it is useful to express all quantities in terms of a renormalized hopping which satisfies its own Dyson equation

$$
\hat{T}^{a, r}\left(\tau, \tau^{\prime}\right)=\hat{t}(\tau) \delta\left(\tau-\tau^{\prime}\right)+\int d \tau_{1} d \tau_{2} \hat{t}(\tau) \hat{g}^{a, r}\left(\tau-\tau_{1}\right) \hat{t}^{\dagger}\left(\tau_{1}\right) \hat{g}^{a, r}\left(\tau_{1}-\tau_{2}\right) \hat{T}^{a, r}\left(\tau_{2}, \tau^{\prime}\right) .
$$

This quantity can be viewed as the total hopping amplitude arising from summing up all processes in which one electron is transferred. Clearly, it is formally equivalent to use a renormalized hopping instead of renormalized propagators as they are linked by relations like $\hat{G}_{L L}\left(\tau, \tau^{\prime}\right) \hat{t}\left(\tau^{\prime}\right)=\int d \tau_{1} \hat{g}_{L}\left(\tau-\tau_{1}\right) \hat{T}_{L R}\left(\tau_{1}, \tau^{\prime}\right)$. The current components can now be expressed in terms of the renormalized hopping elements $\hat{T}_{n m}^{a, r}(\omega)$ as

$$
\begin{aligned}
I_{m}= & \frac{2 e}{h} \int d \omega \sum_{n}\left[\hat{T}_{0 n}^{r} \hat{g}_{n n}^{+-} \hat{T}_{n m}^{r \dagger} \hat{g}_{m m}^{a}-\hat{g}_{00}^{r} \hat{T}_{0 n}^{r} \hat{g}_{n n}^{+-} \hat{T}_{n m}^{r \dagger}\right. \\
& \left.+\hat{g}_{00}^{r} \hat{T}_{0 n}^{a \dagger} \hat{g}_{n n}^{+-} \hat{T}_{n m}^{a}-\hat{T}_{0 n}^{a \dagger} \hat{g}_{n n}^{+-} \hat{T}_{n m}^{a} \hat{g}_{m m}^{a}\right]_{11},
\end{aligned}
$$

where we have eliminated the site indexes $L$ and $R$ in the uncoupled Green functions due to the left-right symmetry of the contact. 
The problem is then reduced to that of the evaluation of the components $\hat{T}_{n m}$. From Eq. (24) it can be shown that the components $\hat{T}_{n m}$ (both retarded and advanced parts) satisfy a set of linear equations of the form

$$
\hat{T}_{n m}=\hat{t}_{n m}+\hat{\epsilon}_{n} \hat{T}_{n m}+\hat{V}_{n, n-2} \hat{T}_{n-2, m}+\hat{V}_{n, n+2} \hat{T}_{n+2, m} .
$$

These equations are mathematically equivalent to those describing the motion of electrons in a tight-binding linear chain with "site energies" , $\hat{\epsilon}_{n}$, and "nearest-neighbor couplings", $\hat{V}_{n, n-2}$ and $\hat{V}_{n, n+2}$. The detailed expression of $\hat{\epsilon}_{n}$ and $\hat{V}_{n, m}$ in terms of the unperturbed Green functions are given in Appendix A. This analogy allows us to obtain the Fourier coefficients $\hat{T}_{n m}$ using standard recursion techniques. One can show (see Appendix A) that the following recursive relation holds

$$
\left\{\begin{array}{l}
\hat{T}_{n+2, m}(\omega)=\hat{z}^{+}\left[\omega+(n-1) \omega_{0}\right] \hat{T}_{n m}(\omega) \quad, n \geq 1 \\
\hat{T}_{n-2, m}(\omega)=\hat{z}^{-}\left[\omega+(n+1) \omega_{0}\right] \hat{T}_{n m}(\omega), n \leq-1
\end{array}\right.
$$

where the transfer matrix $\hat{z}^{ \pm}$satisfy the equation

$$
\hat{z}^{ \pm}(\omega)=\left[\hat{I}-\hat{\epsilon}_{ \pm 3}-\hat{V}_{ \pm 3, \pm 5} \hat{z}^{ \pm}\left(\omega \pm \omega_{0}\right)\right]^{-1} .
$$

Clearly, as the transfer matrix $\hat{z}^{ \pm}$connects consecutive harmonics of $\hat{T}$, it can be viewed as a generating function which introduces the effect of a unitary Andreev reflection process. The problem has been reduced to the calculation of only two matrix coefficients like, for instance, $\hat{T}_{1,0}$ and $\hat{T}_{-1,0}$ as a starting point for the generating Eqs. (27) (see Appendix A for details).

In summary, the basic mathematical difficulty lies in the evaluation of the transfer matrix functions $\hat{z}^{ \pm}$from Eq. (28). Although Eq. (28) looks simple, it is nevertheless hard to solve analytically for arbitrary values of $V$. The analytical results presented in this paper are limited to the $e V / \Delta \rightarrow 0$ and $e V / \Delta \rightarrow \infty$ cases where some simplifying relations hold. For intermediate voltages, an accurate numerical solution of Eq. (28) can be obtained. 


\section{B. Analysis of ac and dc $I-V$ characteristics}

In this subsection we analyze the general features of the $I-V$ characteristics of a S-S contact obtained using our formalism. Let us start by briefly discussing the dc current, $I_{0}(V)$, for different values of the transmission, as shown in Fig. 2. Although the overall qualitative features of these curves have been known since the works of Octavio et al. [27, Zaitsev [24] and Arnold [25], more quantitative and detailed analysis are being reported in recent publications [6,8]. The results of Fig. 2 are in agreement with those reported recently by Averin and Bardas [8] which were obtained using the scattering approach.

Two relevant features of these curves are the subharmonic gap structure for $\mathrm{eV}<2 \Delta$ and the excess current for $e V \gg \Delta$. As can be observed, the subgap structure becomes progressively more pronounced with decreasing transmission. Eventually, when $\alpha \ll 1$ the current steps at positions $e V \sim 2 \Delta / n$ can be clearly resolved. In this limit one can isolate the elementary processes which give rise to these steps. It can be shown that in the tunnel limit the $n$-th step can be calculated as

$$
\delta I_{0}^{(n)}=\lim _{e V \rightarrow 2 \Delta^{+} / n} \frac{8 e}{h} \pi^{2} n t^{2 n} \int_{\Delta}^{n e V-\Delta} d \omega\left[\prod_{i=1}^{n-1}\left|g_{12}(\omega-i e V)\right|^{2}\right] \rho_{11}(\omega) \rho_{22}(\omega-n e V) .
$$

By comparison of Eq. (29) with the expression of $I_{A}$ in Eq. (18) it becomes clear that the steps inside the gap are due to the opening of a new Andreev reflection channel whenever $e V=2 \Delta / n$. Calculation of the integrals in Eq. (29) leads to

$$
\delta I_{0}^{(n)}=\frac{e \Delta \alpha^{n}}{\hbar}\left(\frac{2 n}{4^{2 n-1}}\right)\left(\frac{n^{n}}{n !}\right)^{2}
$$

in agreement with the recent prediction of Bratus et al. [6]. On the other hand, when $\alpha \rightarrow 1$ the subgap structure is completely washed out and there appears an excess current even in the small bias limit.

The other relevant feature of the dc current, namely the large voltage excess current, can be analytically evaluated within our model for any transmission value. The main simplification in this limit comes from the fact that only the lowest order Andreev reflection 
process gives a significant contribution to the excess current 27]. This implies that one can truncate the system of equations (26) for harmonic indexes $n>1$, the resulting simplified system can then be solved explicitly for $\hat{T}_{1,0}(\omega)$ and $\hat{T}_{-1,0}(\omega)$ (for details see Appendix B). As shown in this Appendix the simple result $I_{\text {exc }}^{S S}=2 I_{\text {exc }}^{N S}$ is obtained for any value of the transmission. Although this is the expected physically sound result, to our knowledge, it has not been shown explicitly before except for the ballistic case [24,28. Moreover, some authors have reported the existence of a negative excess current for low transmissions 29 which seems to be in contradiction with the above result. However, one should notice that the excess current as defined above is an asymptotic quantity (only valid in the $e V / \Delta \rightarrow \infty$ limit). When corrections of order $\Delta / e V$ are taken into account one actually can have a defect instead of an excess current for sufficiently low transmission.

The algorithm described in subsection A allows an efficient evaluation of the higher order ac components of the current. For the following analysis we decompose the ac current, Eq. (22), into its dissipative and non-dissipative contributions given respectively by

$$
I_{D}=I_{0}+\sum_{m} I_{m}^{D} \cos \left(m \omega_{0} \tau\right)
$$

and

$$
I_{S}=\sum_{m} I_{m}^{S} \sin \left(m \omega_{0} \tau\right)
$$

where $I_{m}^{D}=2 \operatorname{Re}\left(I_{m}\right)$ and $I_{m}^{S}=-2 \operatorname{Im}\left(I_{m}\right)$.

The results obtained for the first three $I_{m}^{D}$ and $I_{m}^{S}$ components are depicted in Fig. 3 and Fig. 4. As can be observed, these components become exponentially small for bias voltages larger than $\Delta / n$. On the contrary, when $e V<\Delta / n$ the decay of the ac components with increasing $n$ becomes slower. The analysis of the higher order components reveals a decay for $e V<\Delta / n$ close to a inverse power law. As a consequence of this slow decay, one is forced to take an increasing number of ac components into account in order to adequately describe the behavior at small bias. This will be the subject of the next subsection. 


\section{Small bias regime}

In this subsection we concentrate on the $e V / \Delta \rightarrow 0$ case, which turns out to exhibit a remarkable variety of different regimes according to the values of the parameters $\alpha \Delta$ and the inelastic scattering rate $\eta$. As it is well known, the main difficulty for obtaining quantitative results in this limit lies in the fact that the number of MAR contributing to the current grows with decreasing $V$ as $\sim \Delta / e V$ [25]. Furthermore, the amplitudes of these multiple processes do not decay when $(V, \eta) \rightarrow 0$ leading to the appearance of divergencies in the perturbative

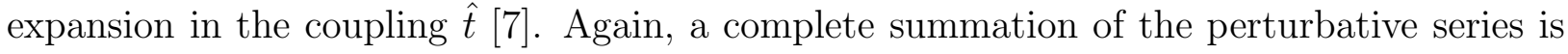
needed in order to regularize these divergencies. An additional difficulty arises, as will be discussed below, from the fact that the limits $V \rightarrow 0$ and $\eta \rightarrow 0$ do not actually commute.

When decreasing the bias voltage two main situations can be reached depending on the strength of the inelastic scattering rate at the superconducting electrodes: the case of $e V / \Delta \rightarrow 0$ and finite $\eta$ has been discussed by the present authors in recent publications [7]; on the other hand, the case of small $V / \Delta$ and negligible $\eta$ has been recently addressed to by Averin and Bardas [8]. In what follows we summarize the main results for both regimes and analyze the conditions for their actual observability in a real SQPC.

Within our formalism, analytical results in the small bias limit become feasible since the transfer matrix $\hat{z}^{ \pm}(\omega)$ tends to a scalar quantity having the form of a simple phase factor inside the gap region. As shown in Appendix $\mathrm{C}$ for $(\eta, V) \rightarrow 0$ we find

$$
\hat{z}^{ \pm}(\omega)=z(\omega)=e^{i \varphi(\omega)}, \quad \Delta \sqrt{1-\alpha} \leq|\omega| \leq \Delta
$$

where

$$
\varphi(\omega)=\arcsin \left(\frac{2}{\alpha \Delta^{2}} \sqrt{\Delta^{2}-\omega^{2}} \sqrt{\omega^{2}-(1-\alpha) \Delta^{2}}\right)
$$

As the multiple Andreev processes are generated by successive applications of $\hat{z}(\omega)$, Eq. (33) indicates that these processes do not decay in this limit inside the gap region. This infinite series of MAR gives rise to the well known bound states spectrum of a currentcarrying SQPC at zero bias voltage [30]. As shown in Appendix C, the positions of these 
bound states are determined by the condition $\varphi(\omega)=\phi$. The presence of a small but finite $\eta$ or $V$ (whichever is larger) introduces an effective damping into the otherwise infinite series of MAR.

When this effective damping is due to a finite $\eta(e V \ll \eta)$ a linear regime can be defined where the total current is given by $I_{S}(\phi)+G(\phi) V, G(\phi)$ being a phase-dependent linear conductance [24,7]. Within this linear regime, one can identify two different sub-regimes according to the ratio $\eta / \alpha \Delta$. The case $\eta / \alpha \Delta \ll 1$ corresponds to a situation where MAR are very weakly damped and give the dominant contribution to the current. The physical picture one can have in mind is that of electrons and holes Andreev-reflecting between the electrodes for a very long time before being inelastically scattered [31]. In order to illustrate the dominant contribution of the processes inside the gap for the weakly damped case, we represent in Fig. 5 the current density corresponding to the $I_{0}$ component for three values of the transmission. Three important features of this linear regime are displayed in this figure: firstly, the current density inside the gap increases as $\sim 1 / \eta$ therefore giving the dominant contribution in the weakly damped case; secondly, there is a region inside the gap of width $2 \Delta \sqrt{1-\alpha}$ in which the current density vanishes. This is the forbidden energy region for bound states at a given transmission. Finally, in Fig. 5 (c) one can observe that the contribution of the continuum outside the gap becomes important as $\alpha<\eta / \Delta$. In the limit $\eta / \alpha \Delta \gg 1$ a second sub-regime is reached where the contributions of MAR are heavily damped and the current is dominated by single quasi-particle tunneling processes. The transition between these two sub-regimes has been analyzed with detail in Refs. [7].

In order to identify the actual sub-regime for a real SQPC an estimation of the order of magnitude of $\eta$ is needed. In Ref. [18] $\eta$ is estimated from the electron-phonon interaction to be a small fraction of the gap for traditional superconductors. Thus, our theory predicts that a real SQPC would generally fall into the weakly damped case except for extremely low transmissions.

For this sub-regime the supercurrent $I_{S}$ and the linear conductance $G(\phi)$ can be obtained analytically as discussed in Appendix C. In particular, for $G(\phi)$ one obtains 


$$
G(\phi)=\frac{2 e^{2}}{h} \frac{\pi}{16 \eta}\left[\frac{\Delta \alpha \sin \phi}{\sqrt{1-\alpha \sin ^{2}(\phi / 2)}} \operatorname{sech}\left(\frac{\beta \omega_{S}}{2}\right)\right]^{2} \beta,
$$

where $\omega_{S}$ is the position of the bound states inside the gap and $\beta=1 / k_{B} T$. This expression for large transmission and small temperatures gives a phase-dependence which is in qualitative agreement with the few available experimental results, performed in non-mesoscopic contacts [32]. The unusual phase-dependence of Eq. (34) which deviates strongly from the $\cos (\phi)$ form predicted by the standard tunnel theory may explain the old controversy between tunnel theory and experiments known as the $\cos (\phi)$ problem [33, 34].

On the other hand, when the truncation of the infinite series of MAR is caused by a finite $V$ (with negligible $\eta$ ), analytical results have only been obtained in the quasi-ballistic limit, i.e. $\alpha \rightarrow 1$. A closer inspection of the $I-V$ curves in the small bias region and for $\alpha \sim 1$ reveals that the supercurrent components decay exponentially from its value at $V=0$ with a collapsing width $\sim(1-\alpha) \Delta$. This is illustrated in Fig. 6 (a) where a blow up of the behavior of $I_{1}^{S}$ for small bias voltages and quasi-ballistic transmissions is shown. In the limit $\alpha \rightarrow 1$ the supercurrent becomes a delta function at $V=0$. On the contrary, the dissipative components in this same limit tend to a finite value outside the region of width $\sim(1-\alpha) \Delta$. This behavior is shown in Fig. 6 (b) where $I_{1}^{D}$ is plotted in the same magnified scale as $I_{1}^{S}$. The summation of these dissipative components for $\alpha=1$ and very small $V$ yields (see Appendix C)

$$
I_{D}(\phi)=\frac{e \Delta}{\hbar}|\sin (\phi / 2)| \operatorname{sign} V,
$$

in agreement with the result recently derived by Averin and Bardas [8]. The existence of a region of decreasing width $V \sim(1-\alpha) \Delta$ in which this crossover from supercurrent to dissipative current takes place can be associated with the collapse of the forbidden region for MAR inside the superconducting gap taking place when $\alpha \rightarrow 1$. In this way, when $V$ is small compared to the width of the forbidden region the excitation of quasi-particles from states at $\omega<-\Delta \sqrt{1-\alpha}$ into states at $\omega>\Delta \sqrt{1-\alpha}$ is negligible and there is no appreciable dissipative current; whereas the opposite situation holds for $V>\sqrt{1-\alpha} \Delta$. 
Averin and Bardas [8] have described this crossover as a Landau-Zener transition in which the non-dissipative and the dissipative components scale with $\alpha$ and $V$ as $(1-p)$ and $p$ respectively, where $p=\exp [-\pi(1-\alpha) \Delta / e V]$. The numerical results for sufficiently small $e V / \Delta$ and $(1-\alpha)$ are well fitted by these scaling laws. However, a careful analysis reveals that their range of validity around $V=0$ and $\alpha=1$ decreases strongly when increasing the component number.

In summary, in this small bias limit one can identify four different sub-regimes depending on the relative values of parameters $\eta, \alpha \Delta$ and $e V$. The prediction of the actual behavior of a real SQPC in this limit would therefore require a careful estimation of all these parameters. In this respect, one should keep in mind that while $e V$ and $\alpha$ can be varied experimentally in a rather controlled way, the inelastic scattering rate $\eta$ is an intrinsic property of the superconducting electrodes much more difficult to control. The unavoidable presence of some degree of inelastic scattering can prevent the actual observability of the crossover from non-dissipative to dissipative behavior described after Eq. (35). The requirement of $e V \sim(1-\alpha) \Delta$ together with that of $\alpha \sim 1$ can actually imply $e V<\eta$ which would rather correspond to the linear regime. Finally, when considering the experimental test of all these theoretical predictions the relevance of noise in a real SQPC should be taken into account. Recent theoretical predictions [35,36] suggest that the magnitude of thermal noise in a single mode superconducting device can be extremely large near ballistic conditions.

\section{CONCLUDING REMARKS}

In the present work we have presented a Hamiltonian approach for describing the transport properties of single mode N-S and S-S contacts.

It has been explicitly demonstrated that this approach is, with some simplifying assumptions, equivalent to the phenomenological scattering approach. We believe that the present work can help clarifying the somewhat recurrent discussion about the unsuitability of a Hamiltonian approach for obtaining the transport properties of a N-S or S-S contact: 
when performing the calculations up to infinite order in the coupling $t$ all the unphysical divergences are eliminated and the results become equivalent to those of scattering theory.

On the other hand, the present approach has been applied to discuss in detail the dc and ac $I-V$ characteristics of a SQPC. In particular, we have concentrated on the less understood small bias voltage limit where one can identify four different sub-regimes depending on the values of the contact transmission and inelastic scattering rate. Finally, we have discussed the conditions for the experimental observability of the theoretical predictions in this limit.

Although in the present work we have restricted the discussion to the simplest singlemode case with an energy-independent transmission coefficient, the general model introduced in section II can describe more complex situations which may be relevant for a closer comparison with recent experiments [5]. Work along this line is under progress.

\section{ACKNOWLEDGMENTS}

Support by Spanish CICYT (Contract No. PB93-0260) is acknowledged. The authors would like to thank A. Lopez Dávalos for his helpful remarks on this work.

\section{APPENDIX A:}

In this Appendix we describe the algorithm for evaluating the ac current components in a S-S biased contact. As it has been pointed out in section IV, we can express the current in terms of the retarded and advanced Fourier components of the renormalized hopping $\hat{T}_{n m}(\omega)$ satisfying Eq. (26)

$$
\hat{T}_{n m}=\hat{t}_{n m}+\hat{\epsilon}_{n} \hat{T}_{n m}+\hat{V}_{n, n-2} \hat{T}_{n-2, m}+\hat{V}_{n, n+2} \hat{T}_{n+2, m}
$$

where the matrix coefficients $\hat{\epsilon}_{n}$ and $\hat{V}_{n m}$ can be expressed in terms of the uncoupled Green functions as

$$
\hat{\epsilon}_{n}=t^{2}\left(\begin{array}{ll}
g_{n+1} g_{n} & g_{n+1} f_{n} \\
g_{n-1} f_{n} & g_{n-1} g_{n}
\end{array}\right)
$$




$$
\begin{aligned}
& \hat{V}_{n, n+2}=-t^{2} f_{n+1}\left(\begin{array}{cc}
f_{n+2} & g_{n+2} \\
0 & 0
\end{array}\right) \\
& \hat{V}_{n, n-2}=-t^{2} f_{n-1}\left(\begin{array}{cc}
0 & 0 \\
g_{n-2} & f_{n-2}
\end{array}\right),
\end{aligned}
$$

where $f(\omega) \equiv g_{12}(\omega)=g_{21}(\omega)$ and $g(\omega) \equiv g_{11}(\omega)=g_{22}(\omega)$ due to electron-hole symmetry. In the above equations the short-hand notation $g_{n}=g\left(\omega+n \omega_{0} / 2\right)$ is used. Moreover, the site indexes in the Green functions have been omitted since we are considering a symmetric contact.

As commented in section IV, the linear Eqs. (A1) are analogous to those describing a tight-binding chain with nearest-neighbor hopping parameters $\hat{V}_{n, n+2}$ and $\hat{V}_{n, n-2}$. A solution can then be obtained by standard recursive techniques. It is straightforward to show that the following recursive relations between the coefficients $\hat{T}_{n m}$ hold

$$
\begin{array}{ll}
\hat{T}_{n+2, m}(\omega)=\hat{z}^{+}\left[\omega+(n-1) \omega_{0}\right] \hat{T}_{n m}(\omega), & n \geq 1 \\
\hat{T}_{n-2, m}(\omega)=\hat{z}^{-}\left[\omega+(n+1) \omega_{0}\right] \hat{T}_{n m}(\omega), & n \leq-1
\end{array}
$$

where the transfer matrix $\hat{z}^{ \pm}(\omega)$ satisfy the equation

$$
\hat{z}^{ \pm}(\omega)=\left[\hat{I}-\hat{\epsilon}_{ \pm 3}-\hat{V}_{ \pm 3, \pm 5} \hat{z}^{ \pm}\left(\omega \pm \omega_{0}\right)\right]^{-1} .
$$

One can see from Eq. (A6) that $\hat{z}^{+}(\omega)$ and $\hat{z}^{-}(\omega)$ are related by $\hat{z}^{-}(\omega, V)=\hat{\sigma}_{x} \hat{z}^{-}(\omega,-V) \hat{\sigma}_{x}$, where $\hat{\sigma}_{x}$ is the corresponding Pauli matrix.

By virtue of the relation $\hat{T}_{n m}(\omega)=\hat{T}_{n-m, 0}\left(\omega+m \omega_{0} / 2\right)$, one can write the current components given by Eq. (25) in terms of $\hat{T}_{n 0}(\omega) \equiv \hat{T}_{n}$. Using recursive relations (A5) the calculation can be reduced to a closed system for coefficients $\hat{T}_{1}$ and $\hat{T}_{-1}$

$$
\begin{array}{r}
{\left[\hat{I}-\hat{\epsilon}_{1}-\hat{V}_{13} \hat{z}^{+}(\omega)\right] \hat{T}_{1}=\hat{t}_{10}+\hat{V}_{1,-1} \hat{T}_{-1}} \\
{\left[\hat{I}-\hat{\epsilon}_{-1}-\hat{V}_{-1,-3} \hat{z}^{-}(\omega)\right] \hat{T}_{-1}=\hat{t}_{-10}+\hat{V}_{-1,1} \hat{T}_{1} .}
\end{array}
$$


The remaining task is the calculation of the transfer matrix $\hat{z}^{+}(\omega)$. It can be shown that the solution of Eq. (A6) is a diagonal matrix whose elements can be expressed in terms of a scalar function $\lambda^{+}(\omega)$

$$
\hat{z}^{+}(\omega)=-t^{2}\left(\begin{array}{cc}
f_{2} f_{3} \frac{\delta_{0}^{+} \delta_{1}^{+}}{\lambda_{0}^{+} \lambda_{1}^{+}} & 0 \\
0 & f_{1} f_{2} \frac{\delta_{-1}^{+} \delta_{0}^{+}}{\lambda_{0}^{+} \lambda_{1}^{+}}
\end{array}\right),
$$

where $\lambda_{n}^{+}=\lambda^{+}\left(\omega+n \omega_{0} / 2\right)$ and $\delta_{n}^{+}=\left(\lambda_{n}^{+}-g_{n+2}\right) / t^{2} f_{n+2}^{2}$. The function $\lambda^{+}(\omega)$ satisfies the following equation

$$
\lambda_{0}^{+}=a+b \lambda_{0}^{+}+c \lambda_{2}^{+}+d \lambda_{0}^{+} \lambda_{2}^{+},
$$

where, taking into account Eq. (17), the coefficients can be written as $a=g_{2}+\left(t^{2} / W^{2}\right) g_{3}$, $b=t^{2} g_{2} g_{3}, c=-t^{2}\left[g_{2} g_{3}-\left(t^{2} / W^{4}\right)\right]$ and $d=t^{2}\left[g_{3}+\left(t^{2} / W^{2}\right) g_{2}\right]$. For an arbitrary bias voltage, Eq. (A9) can only be solved numerically. However, as we show in Appendix B and C it is possible to obtain analytical solutions in the two special cases $\omega_{0} \rightarrow 0$ and $\omega_{0} \rightarrow \infty$.

Once $\hat{z}^{+}(\omega)$ has been determined one can calculate the coefficients $\hat{T}_{1}$ and $\hat{T}_{-1}$ from Eq. (A7)

$$
\begin{gathered}
\hat{T}_{1}=\frac{-t}{1-t^{2} \lambda_{2}^{-} \lambda_{-1}^{+}}\left(\begin{array}{cc}
t^{4} f_{0} f_{1} \delta_{2}^{-} \delta_{-1}^{+} & t^{2} f_{1} \delta_{-1}^{+} \\
t^{2} f_{0} \delta_{2}^{-} & 1
\end{array}\right) \\
\hat{T}_{-1}(\omega, V)=-\hat{\sigma}_{x} \hat{T}_{1}(\omega,-V) \hat{\sigma}_{x},
\end{gathered}
$$

where $\lambda_{n}^{-}(\omega, V)=\lambda_{n}^{+}(\omega,-V)$ and $\delta_{n}^{-}(\omega, V)=\delta_{n}^{+}(\omega,-V)$. The rest of the coefficients $\hat{T}_{n}$ can be calculated from Eqs. (A5)

$$
\begin{aligned}
\hat{T}_{2 n+1} & =\left[\prod_{i=1}^{n} \hat{z}^{+}\left(\omega+(i-1) \omega_{0}\right)\right] \hat{T}_{1}, \quad n>0 \\
\hat{T}_{-n} & =-\hat{\sigma}_{x} \hat{T}_{n}(\omega,-V) \hat{\sigma}_{x}, \quad n>0 .
\end{aligned}
$$

Finally, the current components, separated into its dissipative and non-dissipative parts (Eqs. (31) and (32)) can be calculated from the expressions 


$$
\begin{gathered}
I_{0}=-\frac{4 e}{h} \int_{-\infty}^{\infty} d \omega \sum_{n=o d d} \operatorname{Re}\left\{\operatorname{Tr}\left(\hat{\sigma}_{z} \hat{T}_{n}^{\dagger}(\omega) \hat{g}_{n}^{+-} \hat{T}_{n}(\omega) \hat{g}_{0}^{a}\right)\right\} \\
I_{m}^{D}=-\frac{4 e}{h} \int_{-\infty}^{\infty} d \omega \sum_{n=o d d} \operatorname{Re}\left\{\operatorname{Tr}\left(\hat{\sigma}_{z}\left[\hat{T}_{n+m}^{\dagger}\left(\omega-m \omega_{0} / 2\right)+\hat{T}_{n-m}^{\dagger}\left(\omega+m \omega_{0} / 2\right)\right] \hat{g}_{n}^{+-} \hat{T}_{n}(\omega) \hat{g}_{0}^{a}\right)\right\} \\
I_{m}^{S}=\frac{4 e}{h} \int_{-\infty}^{\infty} d \omega \sum_{n=o d d} \operatorname{Im}\left\{\operatorname{Tr}\left(\hat{\sigma}_{z}\left[\hat{T}_{n+m}^{\dagger}\left(\omega-m \omega_{0} / 2\right)-\hat{T}_{n-m}^{\dagger}\left(\omega+m \omega_{0} / 2\right)\right] \hat{g}_{n}^{+-} \hat{T}_{n}(\omega) \hat{g}_{0}^{a}\right)\right\} .
\end{gathered}
$$

\section{APPENDIX B:}

In this Appendix we give details on the evaluation of the excess current for S-S contacts. In the limit $e V / \Delta \rightarrow \infty$ only the dc current component $I_{0}$ survives. The infinite summation over $n$ in Eq. (A13) can be truncated in this case neglecting the $n>1$ terms. This is justified as the products $f_{n} f_{n+1}$ are negligible in this limit leading to a vanishing transfer matrix $\hat{z}^{ \pm}(\omega)$. Physically, this is equivalent to neglecting multiple Andreev processes for $e V / \Delta \gg 1$. Then, Eq. (A13) reduces to

$$
I_{0} \sim-\frac{4 e}{h} \int_{-\infty}^{\infty} d \omega \sum_{n=-1,1} \operatorname{Re}\left\{\operatorname{Tr}\left(\hat{\sigma}_{z} \hat{T}_{n}^{\dagger}(\omega) \hat{g}_{n}^{+-}(\omega) \hat{T}_{n}(\omega) \hat{g}_{0}^{a}\right)\right\}
$$

with

$$
\begin{aligned}
\hat{T}_{1} & \sim \frac{-t}{1-t^{2} \lambda_{2}^{-} \lambda_{-1}^{+}}\left(\begin{array}{cc}
0 & t^{2} f_{1} \delta_{-1}^{+} \\
t^{2} f_{0} \delta_{2}^{-} & 1
\end{array}\right) \\
\hat{T}_{-1}(\omega, V) & =-\hat{\sigma}_{x} \hat{T}_{1}(\omega,-V) \hat{\sigma}_{x} .
\end{aligned}
$$

On the other hand, when neglecting contributions of order $\Delta / e V$ Eq. (A9) simply yields $\lambda_{n}^{+} \sim\left(g_{n+2}+i t^{2} / W^{3}\right) /\left(1-i t^{2} g_{n+2} / W\right)$. We then obtain from Eq. (B1) the simple result $I_{e x c}^{S S}=2 I_{e x c}^{N N}$, for the excess current at zero temperature and any value of the transmission coefficient. 


\section{APPENDIX C:}

In this Appendix we give the main steps in the analytical calculation of the current components in the limit $\mathrm{eV} / \Delta \rightarrow 0$.

\section{Linear regime $(\eta \gg e V)$}

The small voltage response can be straightforwardly derived from Eqs. (A13-A15) by expanding the Fermi functions appearing in $\hat{g}^{+-}$up to first order in $e V: n_{F}\left(\omega+n \omega_{0} / 2\right) \sim$ $n_{F}(\omega)-(\beta / 8) n \omega_{0} \operatorname{sech}^{2}(\beta \omega / 2)$ and evaluating the rest of these expressions at $e V=0$. The current components can be then written as

$$
\begin{gathered}
I_{0}=\frac{2 e^{2}}{h} \beta V \int_{-\infty}^{\infty} d \omega \operatorname{sech}^{2}\left(\frac{\beta \omega}{2}\right) \sum_{n=o d d>0} n \operatorname{Re}\left\{\operatorname{Tr}\left(\hat{\sigma}_{z} \hat{T}_{n}^{\dagger}\left(\hat{g}_{0}^{a}-\hat{g}_{0}^{r}\right) \hat{T}_{n} \hat{g}_{0}^{a}\right)\right\} \\
I_{m}^{D}=\frac{2 e^{2}}{h} \beta V \int_{-\infty}^{\infty} d \omega \operatorname{sech}^{2}\left(\frac{\beta \omega}{2}\right) \sum_{n=o d d>0} n \operatorname{Re}\left\{\operatorname{Tr}\left(\hat{\sigma}_{z}\left[\hat{T}_{n+m}^{\dagger}+\hat{T}_{n-m}^{\dagger}\right]\left(\hat{g}_{0}^{a}-\hat{g}_{0}^{r}\right) \hat{T}_{n} \hat{g}_{0}^{a}\right)\right\} \\
I_{m}^{S}=\frac{8 e}{h} \int_{-\infty}^{\infty} d \omega n_{F}(\omega) \sum_{n=o d d>0} \operatorname{Im}\left\{\operatorname{Tr}\left(\hat{\sigma}_{z}\left[\hat{T}_{n+m}^{\dagger}-\hat{T}_{n-m}^{\dagger}\right]\left(\hat{g}_{0}^{a}-\hat{g}_{0}^{r}\right) \hat{T}_{n} \hat{g}_{0}^{a}\right)\right\} .
\end{gathered}
$$

Thus, the dissipative contribution, $I_{D}$, goes to zero as $I_{D}(\phi) \sim G(\phi) V, G(\phi)$ being the phase-dependent linear conductance, while the supercurrent part, $I_{S}(\phi)$, tends to a finite value at $V=0$.

In the zero voltage limit the coefficients $\hat{T}_{n}$ adopt a simple form. The transfer matrix $\hat{z}^{ \pm}(\omega)$ becomes a scalar function: $\hat{z}^{+}(\omega)=\hat{z}^{-}(\omega) \equiv z(\omega) \hat{I}$; with $z(\omega)=-t^{2} f \delta^{2} / \lambda^{2}$, where $\lambda^{+}(\omega)=\lambda^{-}(\omega) \equiv \lambda(\omega)$ satisfies the simple quadratic equation

$$
t^{2} g(\omega) \lambda^{2}(\omega)-\left(1-\frac{t^{2}}{W^{2}}\right) \lambda(w)+g(\omega)=0
$$

Finally, the coefficients $\hat{T}_{n}$ adopt the form

$$
\hat{T}_{1}(\omega)=\frac{-t}{1-t^{2} \lambda^{2}}\left(\begin{array}{cc}
t^{4} f^{2} \delta^{2} & t^{2} f \delta \\
t^{2} f \delta & 1
\end{array}\right)
$$




$$
\hat{T}_{2 n+1}(\omega)=z^{n}(\omega) \hat{T}_{1}(\omega), \quad n \geq 0
$$

Due to these simple recursive relations, the series appearing in the current components become geometrical series, which can be summed up without difficulty. In the weakly damped case, $\eta / \alpha \Delta \ll 1$, these summations lead to analytical expressions for the dissipative and non-dissipative parts of the current. By solving Eq. (C4) up to corrections of order $\eta / \alpha \Delta$ one obtains

$$
z(\omega)=e^{i \varphi(\omega)}-\frac{4 \omega \eta}{\alpha \Delta^{2}}[i+\operatorname{cotg}(\varphi(\omega))], \quad \Delta \sqrt{1-\alpha} \leq|\omega| \leq \Delta,
$$

where

$$
\varphi(\omega)=\arcsin \left(\frac{2}{\alpha \Delta^{2}} \sqrt{\Delta^{2}-\omega^{2}} \sqrt{\omega^{2}-(1-\alpha) \Delta^{2}}\right)
$$

The summation of the geometrical series yield

$$
\begin{gathered}
I_{0}=\frac{2 e^{2}}{h} \beta V \int_{-\infty}^{\infty} d \omega \operatorname{sech}^{2}\left(\frac{\beta \omega}{2}\right) \operatorname{Re}\{A(\omega)\} \frac{1+|z|^{2}}{\left(1-|z|^{2}\right)^{2}} \\
I_{m}^{D}=\frac{2 e^{2}}{h} \beta V \int_{-\infty}^{\infty} d \omega \operatorname{sech}^{2}\left(\frac{\beta \omega}{2}\right) \operatorname{Re}\left\{A(\omega)\left[\left(z^{m}+\left(z^{*}\right)^{m}\right) \frac{1+|z|^{2}}{\left(1-|z|^{2}\right)^{2}}+\frac{2 m z^{m}}{1-|z|^{2}}\right]\right\} \\
I_{m}^{S}=\frac{8 e}{h} \int_{-\infty}^{\infty} d \omega n_{F}(\omega) \operatorname{Im}\left\{A(\omega) \frac{\left(z^{*}\right)^{m}-z^{m}}{1-|z|^{2}}\right\},
\end{gathered}
$$

where $A(\omega) \equiv \operatorname{Tr}\left[\hat{\sigma}_{z} \hat{T}_{1}^{\dagger}\left(\hat{g}^{a}-\hat{g}^{r}\right) \hat{T}_{1} \hat{g}^{a}\right]$. It can be noticed that in this weakly damped limit the integrands goes like $1 / \eta$ and the energy interval $\Delta \sqrt{1-\alpha} \leq|\omega| \leq \Delta$ give the main contribution to the current.

Finally, when summing up all ac components to obtain the total dissipative and nondissipative parts, the current densities become singular at the condition $\varphi(\omega)=\phi$. This condition is satisfied for $\omega=\omega_{S}= \pm \Delta \sqrt{1-\alpha \sin ^{2}(\phi / 2)}$ (i.e. at the bound states energy levels) leading to

$$
I_{D}(\phi)=\frac{e^{2} \alpha^{2} \Delta^{4}}{8 \eta h} \beta V \int_{-\infty}^{\infty} d \omega \operatorname{sech}^{2}\left(\frac{\beta \omega}{2}\right) \frac{\sin ^{2} \varphi(\omega)}{\omega} \operatorname{Im}\left\{\frac{1}{\left(\omega-\left|\omega_{S}\right|-i \eta\right)\left(\omega+\left|\omega_{S}\right|-i \eta\right)}\right\}
$$




$$
I_{S}(\phi)=-\frac{2 e}{h} \alpha \Delta^{2} \sin \phi \int_{-\infty}^{\infty} d \omega n_{F}(\omega) \operatorname{Im}\left\{\frac{1}{\left(\omega-\left|\omega_{S}\right|-i \eta\right)\left(\omega+\left|\omega_{S}\right|-i \eta\right)}\right\} .
$$

These integrals can be straightforwardly evaluated. Eq. (C11) gives the expression for the phase-dependent linear conductance given in section IV (Eq. (34)), while Eq. (C12) yields the well known expression for the supercurrent in a single mode SQPC [13, 37

$$
I_{S}(\phi)=\frac{e \Delta^{2} \alpha}{2 \hbar} \frac{\sin (\phi)}{\left|\omega_{S}(\phi)\right|} \tanh \left(\frac{\beta\left|\omega_{S}(\phi)\right|}{2}\right) .
$$

\section{Non-linear regime $(\eta \ll e V)$}

We first rewrite Eqs. (A13-A15) for the current components as

$$
\begin{aligned}
& I_{0}=\frac{4 e}{h} \int_{-\infty}^{\infty} d \omega \tanh \left(\frac{\beta \omega}{2}\right) \sum_{n=o d d>0} \operatorname{Re}\left\{\operatorname{Tr}\left(\hat{\sigma}_{z} \hat{T}_{n}^{\dagger}\left(\hat{g}_{0}^{a}-\hat{g}_{0}^{r}\right) \hat{T}_{n} \hat{g}_{-n}^{a}\right)\right\} \\
& I_{m}^{D}=\frac{4 e}{h} \int_{-\infty}^{\infty} d \omega \tanh \left(\frac{\beta \omega}{2}\right) \sum_{n=o d d>0} \operatorname{Re}\left\{\operatorname{Tr}\left(\hat{\sigma}_{z}\left[\hat{T}_{n+m}^{\dagger} \hat{T}_{n-m}^{\dagger}\right]\left(\hat{g}_{0}^{a}-\hat{g}_{0}^{r}\right) \hat{T}_{n} \hat{g}_{-n}^{a}\right)\right\} \\
& I_{m}^{S}=-\frac{4 e}{h} \int_{-\infty}^{\infty} d \omega \tanh \left(\frac{\beta \omega}{2}\right) \sum_{n=o d d>0} \operatorname{Im}\left\{\operatorname{Tr}\left(\hat{\sigma}_{z}\left[\hat{T}_{n+m}^{\dagger}-\hat{T}_{n-m}^{\dagger}\right]\left(\hat{g}_{0}^{a}-\hat{g}_{0}^{r}\right) \hat{T}_{n} \hat{g}_{-n}^{a}\right)\right\},
\end{aligned}
$$

where a rigid shift of $n \omega_{0} / 2$ in the energy arguments of the different $\hat{T}_{n}$ with respect to the ones appearing in Eq. (A13-A15) has been introduced.

In the limit $\mathrm{eV} \rightarrow 0$, the solution of Eq. (A9) is $\lambda_{n}^{+}=\lambda\left[\omega+(n+2) \omega_{0} / 2\right]$, where $\lambda(\omega)$ satisfies the quadratic Eq. (C4). The coefficients $\hat{T}_{n}(n>0)$ can then be generated starting form $\hat{T}_{1}$ and using the transfer matrix $\hat{z}^{+}(\omega)$. These quantities are obtained from Eqs. (A8) and (A10) making use of the $e V \rightarrow 0$ solution for $\lambda_{n}^{+}$.

The resulting expressions simplify considerably in the ballistic limit where $\lambda_{n}^{+}=i / t$. For energies inside the gap one obtains

$$
\hat{T}_{n}(\omega)=-\frac{t}{2} \prod_{j=1}^{n-1} e^{i \alpha_{j}}\left(\begin{array}{cc}
e^{i\left(\alpha_{0}+\alpha_{n}\right)} & e^{i \alpha_{n}} \\
e^{i \alpha_{0}} & 1
\end{array}\right),
$$


where $\alpha_{n}=\arccos [(\omega+n e V) / \Delta]$. As discussed in Ref. [8], when written as in Eq. (C14), the main contribution to the current in this limit comes from a small energy range around the gap edges. Evaluation of these integrals leads directly to Eq. (35). 


\section{REFERENCES}

[1] A.F. Andreev, Zh. Ekps. Teor. Fiz. 46, 1823 (1964) [ Sov. Phys. JETP 19, 1228 (1964)]; 49, 655 (1966) [22, 455 (1966)]; B.D. Josephson, Phys. Lett. 1, 251 (1962); Rev. Mod. Phys. 36, 216 (1964).

[2] K.K. Likharev, Rev. Mod. Phys. 51, 101 (1979).

[3] G.E. Blonder, M. Tinkham and T.M. Klapwijk, Phys. Rev. B 25, 4515 (1982).

[4] N. van der Post, E.T. Peters, I.K. Yanson and J.M. van Ruitenbeek, Phys. Rev. Lett. 73, 2611 (1994); B.J. Vleeming, C.J. Muller, M.C. Koops, and R. de Bruyn Ouboter, Phys. Rev. B 50, 16741 (1994).

[5] H. Takayanagi, T. Akazaki and J. Nitta, Phys. Rev. Lett. 75, 3533 (1995).

[6] E.N. Bratus, V.S. Shumeiko and G. Wendin, Phys. Rev. Lett. 74, 2110 (1995).

[7] A. Martín-Rodero, A. Levy Yeyati and J.C. Cuevas, Physica B 218, 126 (1996); A. Levy Yeyati, A. Martín-Rodero and J.C. Cuevas, J. Phys.: Condens. Matter 8, 449 (1996).

[8] D. Averin and A. Bardas, Phys. Rev. Lett. 75, 1831 (1995).

[9] R. Landauer, Phil. Mag. 21, 863 (1970).

[10] J. Bardeen, Phys. Rev. Lett. 6, 57 (1961).

[11] V. Ambegaokar and A. Baratoff, Phys. Rev. Lett. 10, 486 (1963); 11, 104 (1963).

[12] J.R. Schrieffer and J.W. Wilkins, Phys. Rev. Lett. 10, 17 (1963); J.W. Wilkins Tunneling Phenomena in Solids, p. 333 (New York, Plenum, 1963).

[13] A. Martín-Rodero, F. J. García-Vidal and A. Levy Yeyati, Phys. Rev. Lett. 72, 554 (1994); A. Levy Yeyati, A. Martín-Rodero, F. J. García-Vidal, Phys. Rev. B 51, 3743 (1995).

[14] A. Levy Yeyati, A. Martín-Rodero and F. Flores, Phys. Rev. Lett. 71, 2991 (1993). 
[15] P.G. de Gennes, Superconductivity of Metals and Alloys (Benjamin, New York, 1966).

[16] D. Rogovin and D.J. Scalapino, Annals of Physics 86, 1 (1974).

[17] L.V. Keldysh, Sov. Phys. JETP 20, 1018 (1965).

[18] A typical estimate of $\eta$ for a traditional superconductor is $\eta / \Delta \sim 10^{-2}$. See for instance, S.B. Kaplan et al., Phys. Rev. B 14, 4854 (1976).

[19] C. Caroli, R. Combescot, P. Nozières and D. Saint-James, J. Phys. C 4, 916 (1971).

[20] J. Ferrer, A. Martín-Rodero and F. Flores, Phys. Rev. B 38, 10113 (1988).

[21] G.D. Mahan Many-Particle Physics (Plenum Press, New York, 1981).

[22] A finite order perturbative calculation in $t$ yields a conductance which diverges at $e V=$ $\Delta$ due to the divergencies of the uncoupled BCS Green functions (Eq. (7)).

[23] L.E. Hasselberg, J. of Phys. F 3, 1438 (1973); L.E. Hasselberg, M.T. Levinsen and M.R. Samuelsen, Phys. Rev. B 9, 3757 (1974).

[24] A.V. Zaitsev, Zh. Eksp. Teor. Fiz. 78, 221 (1980) [Sov. Phys. JETP 51, 111 (1980)].

[25] G. B. Arnold, J. Low Temp. Phys. 59, 143 (1985); J. Low. Temp. Phys. 68, 1 (1987).

[26] A. Levy Yeyati and F. Flores, J. Phys.: Condens. Matter 4, 7341 (1992); A. Levy Yeyati, J. C. Cuevas and A. Martín-Rodero in Photons and local probes, edited by O. Marti and R. Möller, (Kluwer Academic Publishers, 1995).

[27] M. Octavio, M. Tinkham, G.E. Blonder and T.M. Klapwijk, Phys. Rev. B 27, 6739 (1983).

[28] T.M. Klapwijk, G.E. Blonder and M. Tinkham, Physica 109-110 B\&C, 1657 (1982).

[29] K. Flensberg, J. Bindslev Hansen and M. Octavio, Phys. Rev. B 38, 8707 (1988).

[30] A. Furusaki and M. Tsukada, Physica B 165+166, 967 (1990). 
[31] The dominant contribution of MAR in the linear conductance of S-N-S weak links has been recently pointed out by U. Gunsenheimer and A.D. Zaikin, Phys. Rev. B 50, 6317 (1994).

[32] R. Rifkin and B.S. Deaver, Jr., Phys. Rev. B 13, 3894 (1976).

[33] A.B. Zorin et al., Fiz. Nizk. Temp. 5, 1138 (1979) [Sov. J. Low Temp. Phys. 5, 537 (1979)].

[34] A. Barone and G. Paterno, Physics and Applications of the Josephson Effect (Wiley, New York, 1982).

[35] A. Martín-Rodero, A. Levy Yeyati and F.J. García-Vidal, Phys. Rev. B (RC), in press.

[36] R. de Bruyn Ouboter and A.N. Omelyanchouk, Physica B 216, 37 (1995).

[37] C.W. Bennakker in Proceedings of the 14th Taniguchi International Symposium on Transport Phenomena in Mesoscopic Systems, edited by H. Fukuyama and T. Ando (Springer, Berlin 1992). 


\section{FIGURES}

FIG. 1. Schematic representations of the two different situations discussed in Section II.

FIG. 2. The dc current-voltage characteristic of a S-S contact for different values of the normal transmission at zero temperature.

FIG. 3. The first three ac components of the dissipative current for different values of the normal transmission at zero temperature.

FIG. 4. Same as Fig. 4 for the non-dissipative current.

FIG. 5. Current density for the dc component $I_{0}$ within the linear regime discussed in section IV.C. Cases (a), (b) and (c) correspond to transmission values $\alpha=1,0.65$ and 0.04 respectively. In all cases the full line corresponds to $\eta / \Delta=1 / 10$, the dotted line to $\eta / \Delta=1 / 25$ and the broken

line to $\eta / \Delta=1 / 100$. The thermal factor $\operatorname{sech}^{2}(\beta \omega / 2)$ (see Eq. (C8)) has been extracted from the current density.

FIG. 6. Behavior of the first non-dissipative (a) and dissipative (b) ac components in the very small voltage range close to ballistic conditions. These results have been obtained for negligible $\eta$. 


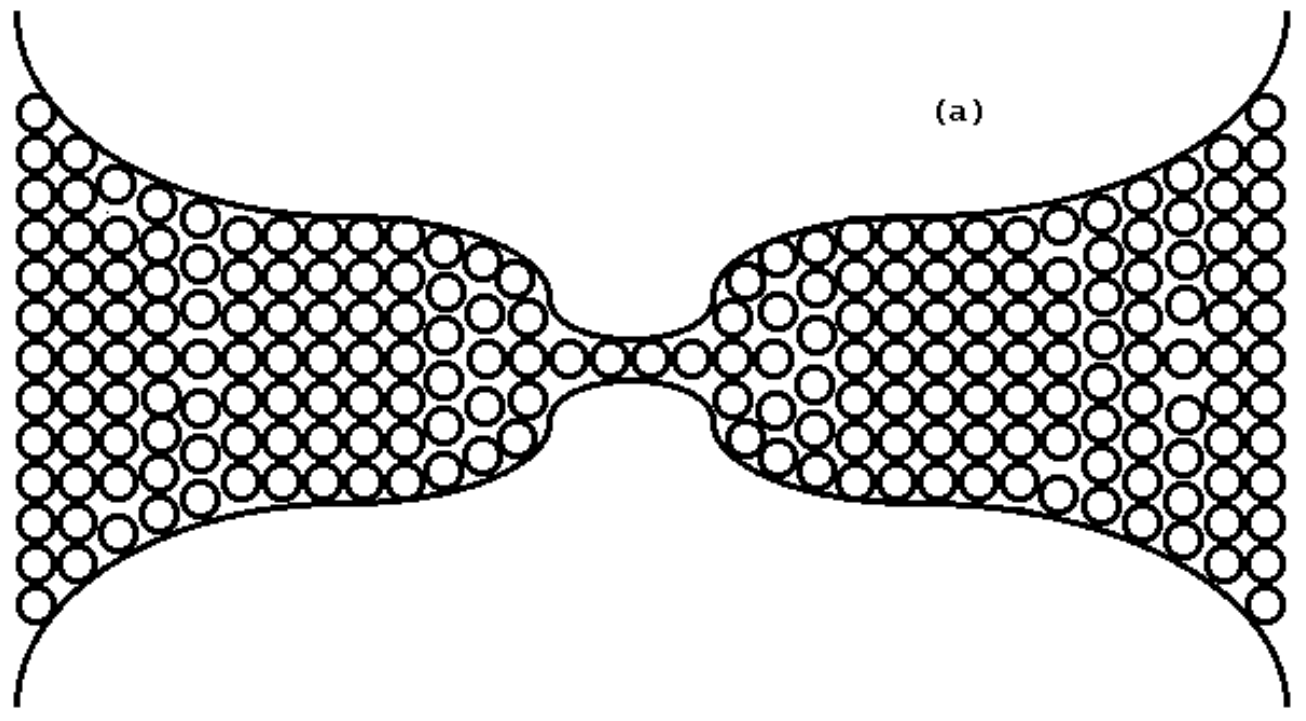

(b)

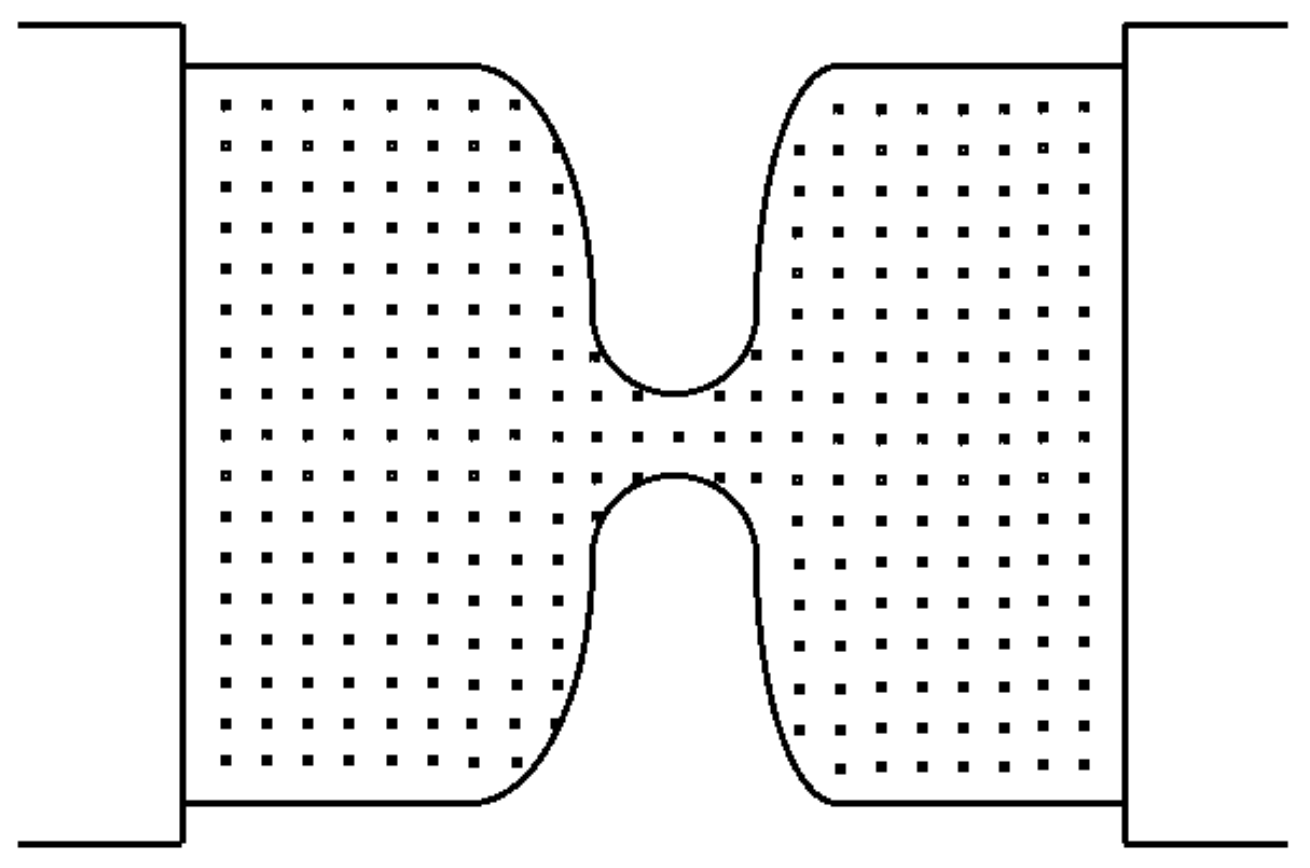




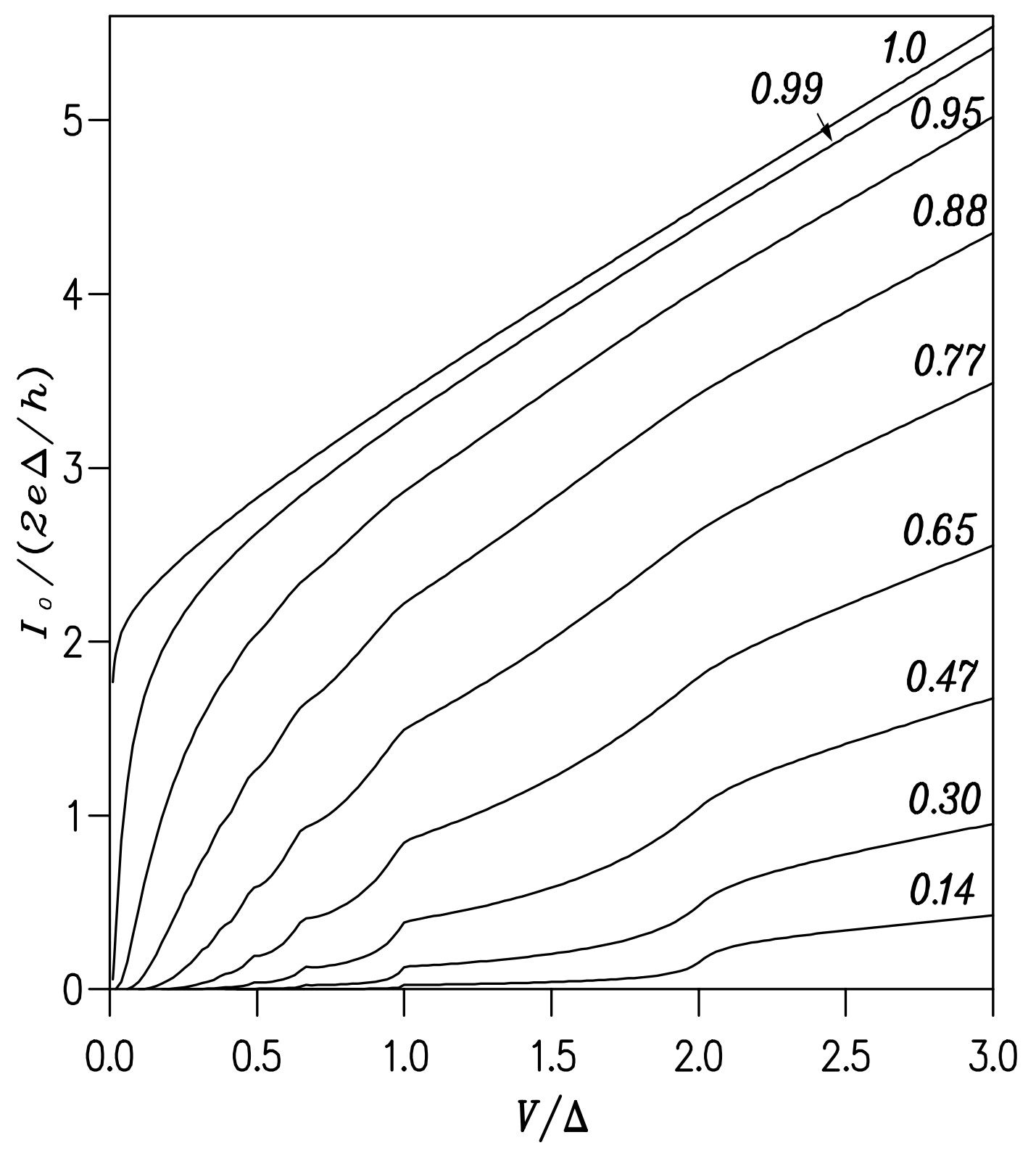




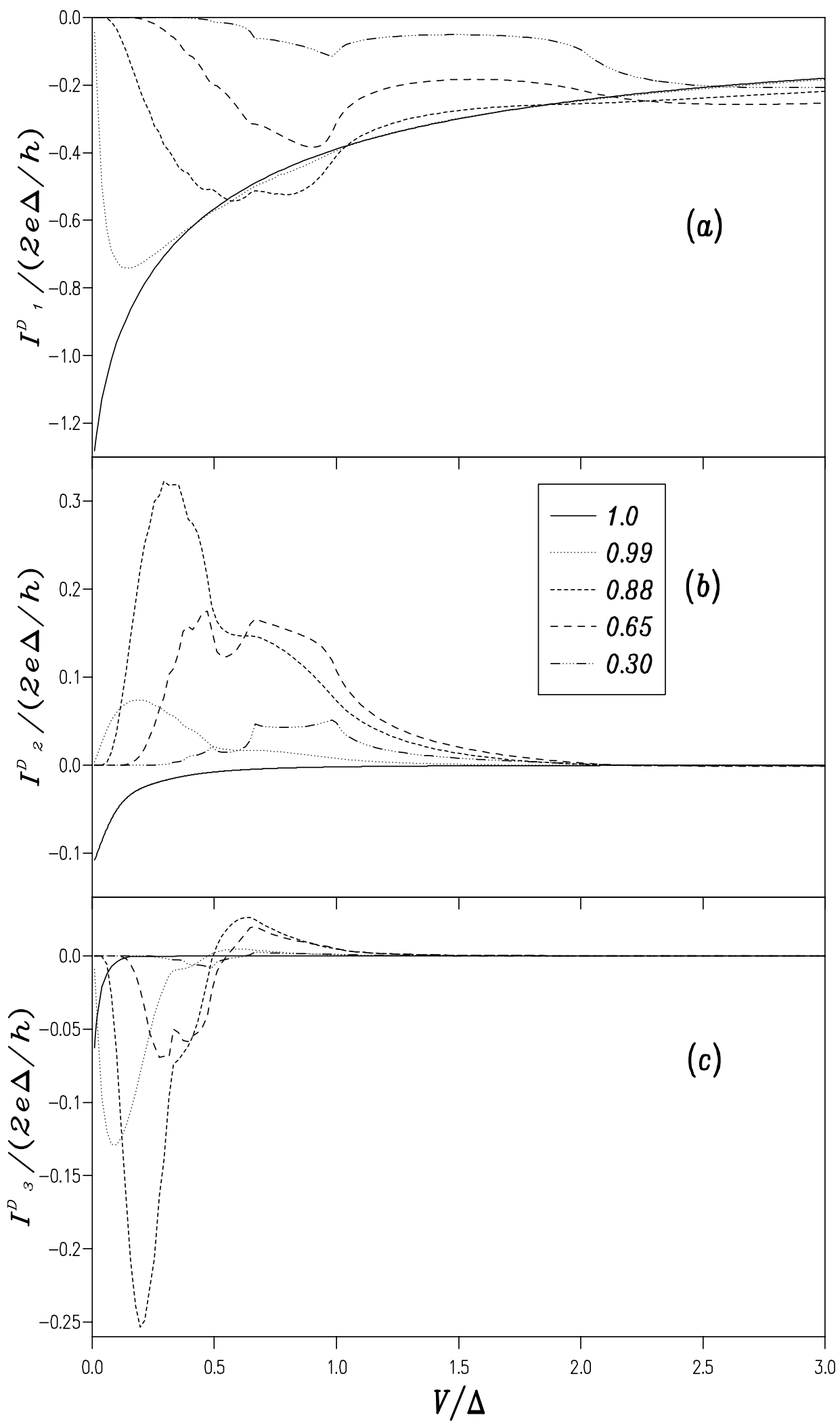




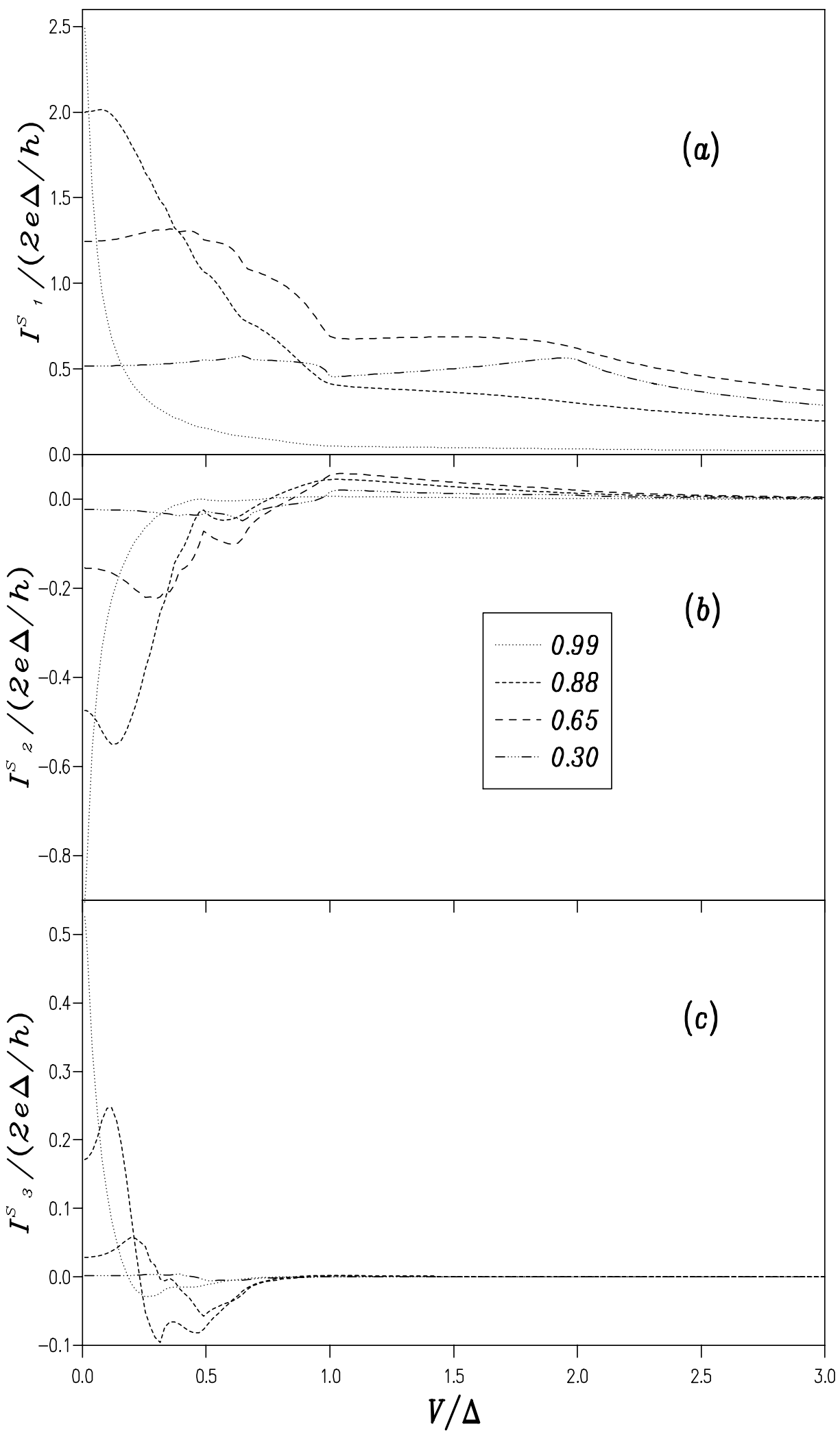




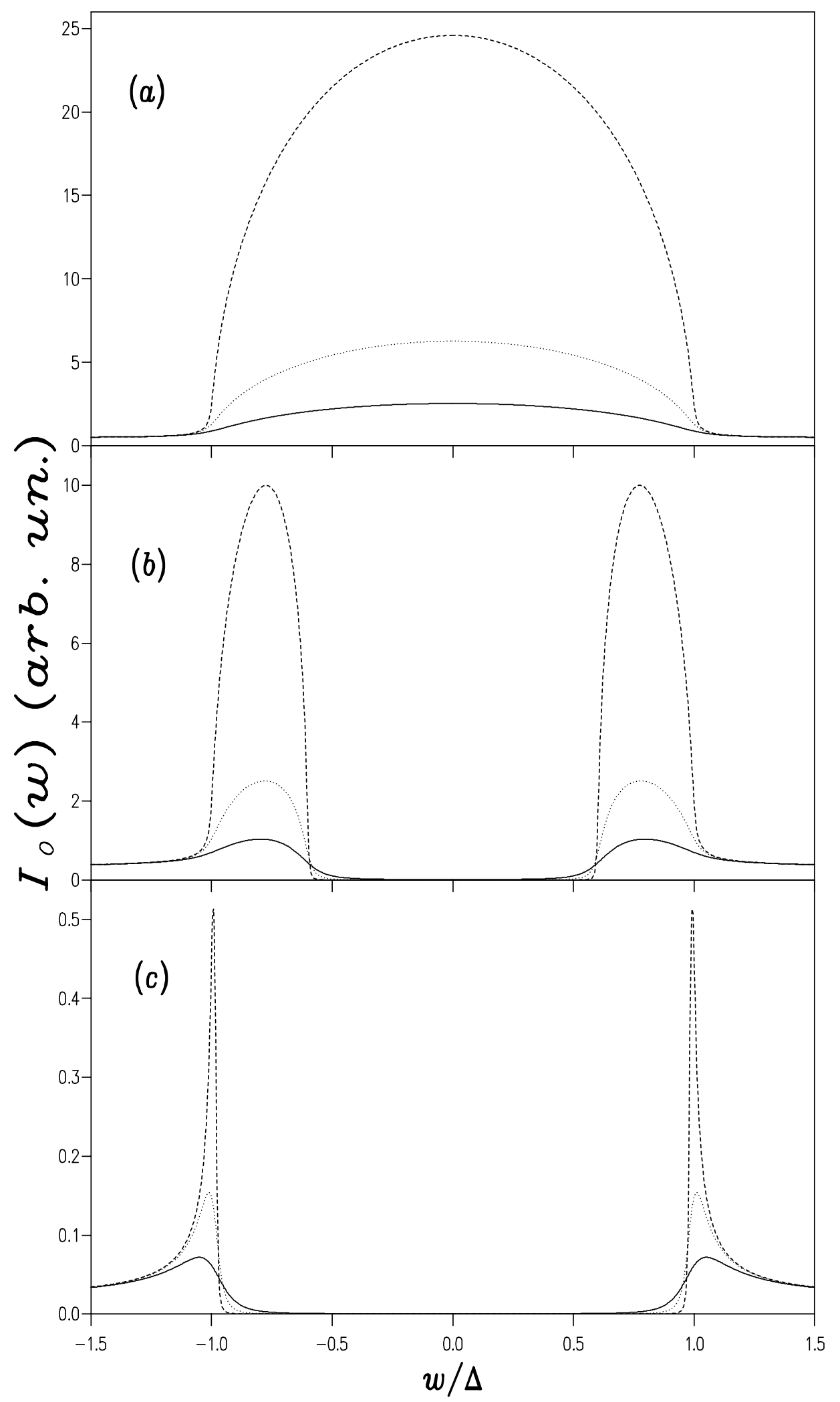




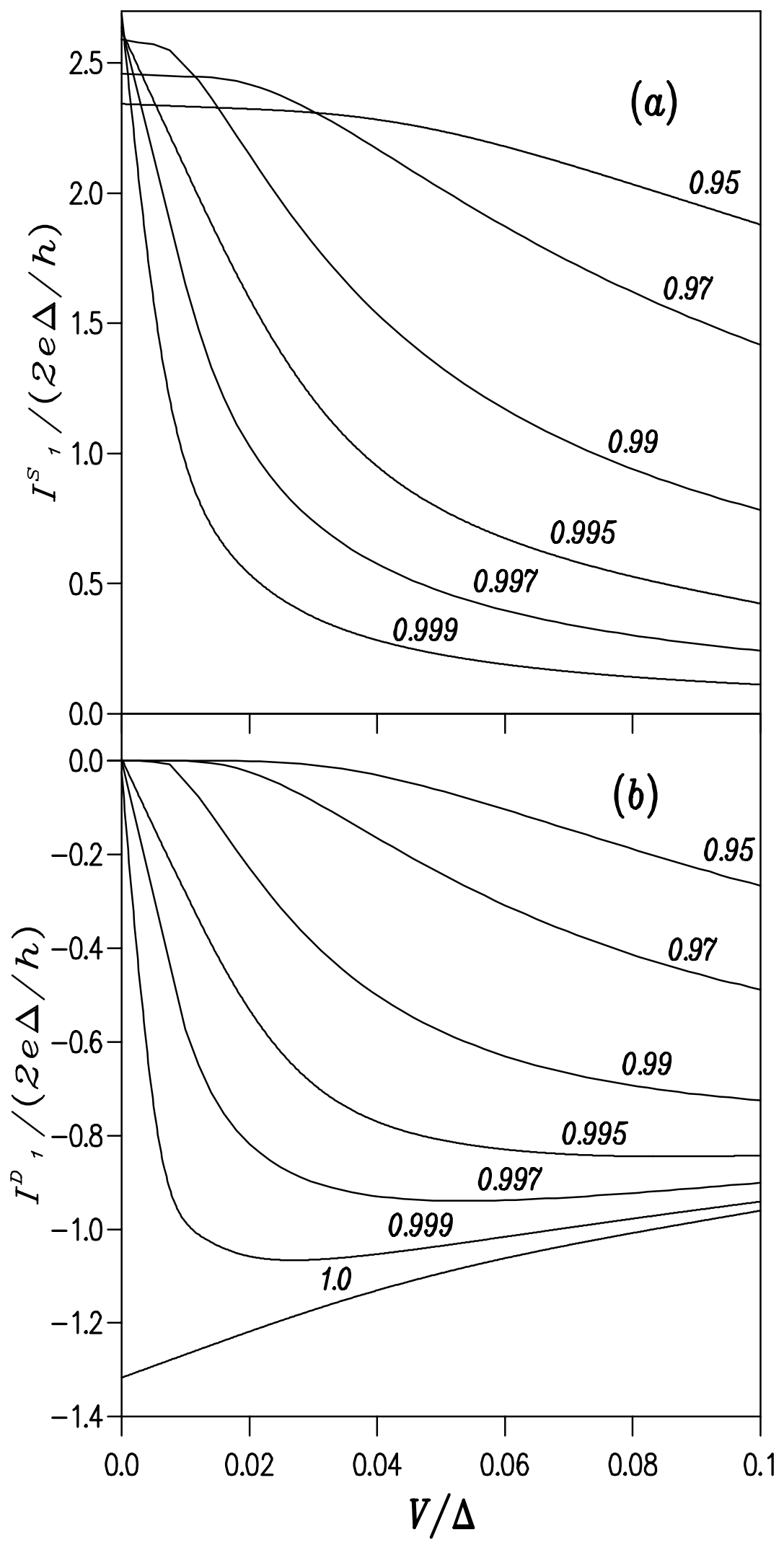

